\title{
PREVALÊNCIA E FATORES ASSOCIADOS À SOBRECARGA DE TRABALHO DE CUIDADORES DE IDOSOS RESTRITOS AO LAR
}

\author{
PREVALENCE OF WORK OVERBURDEN AND ASSOCIATED FACTORS \\ IN CAREGIVERS OF HOMEBOUND ELDERLY
}

\author{
Gracielle Pampolim \\ Curso de Fisioterapia e Medicina \\ Escola Superior de Ciências da Santa Casa \\ de Misericórdia de Vitória - EMESCAM \\ Marise Cupertino Lessa Ferreira \\ Curso de Enfermagem \\ Escola Superior de Ciências da Santa Casa \\ de Misericórdia de Vitória - EMESCAM

\section{Vanezia Gonçalves da Silva} \\ Curso de Medicina \\ Escola Superior de Ciências da Santa Casa \\ de Misericórdia de Vitória - EMESCAM
}

\author{
Luciana Carrupt Machado Sogame \\ Programa de Pós-Graduação em Políticas \\ Públicas e Desenvolvimento Local \\ Escola Superior de Ciências da Santa Casa \\ de Misericórdia de Vitória - EMESCAM \\ Contato \\ Gracielle Pampolim \\ Rua Augusto dos Anjos, 205/501 \\ Vitória - ES \\ E-mail: graciellepampolim@hotmail.com
}

\section{RESUMO}

Introdução: $O$ ato de cuidar da pessoa idosa deve incluir um conjunto de atividades desenvolvidas pela família, comunidade e profissionais de saúde. Objetivo: verificar a presença de sobrecarga de trabalho nos cuidadores de idosos restritos ao lar e a relação entre essa sobrecarga e as características sociodemográficas dos idosos assistidos por uma Unidade de Saúde da Família do município de Vitória-ES. Métodos: Estudo transversal com 165 cuidadores de idosos restritos ao lar assistidos por uma Unidade de Saúde da Família de Vitória-ES. A sobrecarga foi avaliada por meio da Zarit Burden Interview, e coletadas variáveis referentes ao perfil dos idosos. Utilizaram-se os testes Chi-quadrado de Pearson e a Correlação de Spearman para as análises de dados. Resultados: A prevalência de sobrecarga entre os cuidadores foi de $32,7 \%$. Foi identificado que idosos mais jovens e de raça negra/parda sobrecarregam mais seus cuidadores, enquanto a sobrecarga é menor entre os cuidadores de idosos que residem em bairros nobres. Conclusão: Acredita-se que os resultados apresentados neste estudo podem servir como base de avaliação diagnóstica, ressaltando a necessidade de enxergar o cuidador como sujeito que também necessita ser cuidado.

Palavras-chave: Cuidador. Idosos. Saúde da família. 
Introduction: The act of caring for the elderly should include a set of activities developed by the family, community and health professionals. Objective: to verify the presence of work overload in caregivers of the elderly restricted to the home and the relationship between that overload and the socio-demographic characteristics of the elderly, assisted by a Family Health Unit in the city of Vitória-ES. Methods: A crosssectional study was carried out with 165 caregivers of the homebound elderly assisted by a Family Health Unit of Vitória-ES. The burden was assessed through the Zarit Burden Interview, and variables were collected regarding the profile of the elderly. Pearson's Chi-square tests and Spearman's correlation were used for data analyses. Results: The prevalence of overburden among caregivers was $32.7 \%$. Younger and black / brown elderly have been found to overburden more their caregivers, while the burden is lower among caregivers of older people living in noble neighborhoods. Conclusion: It is believed that the results presented in this study can serve as a basis for diagnostic evaluation, highlighting the need to see the caregiver as a subject who also needs to be cared for.

Keywords: Caregivers; Elderly; Family Health

\section{INTRODUÇÃo}

O envelhecimento populacional é uma realidade mundial que vem afetando cada vez mais os países em desenvolvimento ${ }^{1}$. No Brasil, o Instituto Brasileiro de Geografia e Estatística (IBGE) registrou, em 2010, uma importante aceleração do processo de envelhecimento, em que a expectativa de vida da população saltou de 48,0 anos, em 1960, para 73,4 anos, em 2010, e continua crescendo continuamente ${ }^{2}$. Tal fato também é observado em diversos estados, como é o caso do Espírito Santo, onde, em 2004, a cada 100 jovens capixabas, existiam pouco mais de 34 idosos, já em 2014, essa relação quase dobrou, sendo 63,4 idosos para cada 100 jovens $^{3}$.

Caracterizado por um processo normal, gradual e irreversível, o envelhecimento traz consigo uma progressiva redução das capacidades funcionais do organismo ${ }^{4}$, que resultam em redução da independência funcional e possível afastamento social do idoso - por vezes restringindo-o unicamente ao convívio familiar -, podendo ou não estar associado a doenças crônicas. Esse processo faz com que o idoso necessite de apoio e ajuda de terceiros para realização das suas atividades de vida diária, sendo o provedor desse apoio, nos dias atuais, denominado como cuidador ${ }^{5}$.

No entanto, o ato de cuidar da pessoa idosa deve incluir um conjunto de atividades desenvolvidas pela família, comunidade e profissionais de saúde, que se reverte em um ambiente caracterizado pela preocupação com a integralidade, singularidade, valorização e respeito ao outro. Todas essas ações devem ser conduzidas para a identificação das necessidades do idoso, bem como para suporte e apoio ao cuidador evitando a sobrecarga de trabalho6,7.

A sobrecarga de trabalho ocorre quando existe um desequilíbrio da relação funcional entre as exigências do trabalho; e capacidades biológicas e psicológicas do trabalhador, por isso avaliar a carga de trabalho é uma forma de evitar possíveis agravos na saúde do trabalhador, ou seja, daquele que cuida ${ }^{8}$. Apesar de os estudos indicarem a indiscutível presença de sobrecarga nos cuidadores de idosos ${ }^{9-11}$, não foi identificado na literatura um consenso sobre a prevalência desta. Especialmente sem a especificidade de um grupo de idosos dependentes ou com alguma doença crônica. 
Dessa forma definiu-se como objetivo deste estudo verificar a presença de sobrecarga de trabalho nos cuidadores de idosos restritos ao lar e a relação entre essa sobrecarga e as características sócio demográficas dos idosos, assistidos por uma Unidade de Saúde da Família (USF) do município de Vitória-ES.

\section{MÉTODOS}

Trata-se de uma análise secundária do banco de dados do estudo transversal de abordagem quantitativa denominado "Perfil sócio demográfico e de saúde dos idosos restritos ao lar e acamados de uma unidade de saúde da família do Município de Vitória- ES", coletados a partir da pesquisa emanada do Programa Nacional de Reorientação da Formação Profissional em Saúde e Programa de Ensino pelo Trabalho para a Saúde (PRO-PET Saúde), vinculado à Escola Superior de Ciências da Santa Casa de Misericórdia de Vitória (EMESCAM).

\section{Amostragem}

Para composição da amostra do estudo macro, foram considerados 298 idosos restritos ao lar, adscritos à Unidade de Saúde Dr. José Moysés, tendo como base fevereiro de 2014. Destes 298, 215 foram incluídos no estudo, sendo que 83 idosos foram excluídos em razão de óbito, mudança de endereço ou não consentimento para o estudo. Dos incluídos, 165 possuíam cuidadores que responderam ao questionário de sobrecarga. Dessa forma, o presente estudo apresenta uma amostra de conveniência de 165 cuidadores de idosos restritos ao lar.

Foi considerado restrito ao lar o idoso incapaz de sair de casa sem acompanhante ${ }^{12}$; e para classificação destes, utilizaram-se os dados obtidos na ficha de cadastro na Estratégia Saúde da Família registrada na Rede Bem Estar, prontuário eletrônico da rede de atenção do município de Vitória-ES e informações dos Agentes Comunitários de Saúde.

Foi considerado cuidador o indivíduo que desempenha o ato de cuidar da pessoa idosa, zelando pelo bem-estar, saúde, alimentação, higiene pessoal, recreação e lazer, sendo este alguém da família, da comunidade ou de instituições especializadas que exercem o cuidado com ou sem remuneração6 .

Este trabalho foi aprovado pelo Comitê de Ética em Pesquisa da EMESCAM sob o protocolo número 567.990. Os participantes aceitaram participar do estudo mediante assinatura do Termo de Consentimento Livre e Esclarecido, e foram respeitadas todas as diretrizes da Resolução 466/12.

\section{Variáveis Estudadas}

Os dados foram obtidos por meio de entrevista face a face, realizadas na residência dos idosos por acadêmicos participantes do PET dos cursos de fisioterapia, enfermagem e medicina da EMESCAM, previamente treinados pela pesquisadora principal.

A presença de sobrecarga de cuidadores foi avaliada por meio da escala Zarit Burden Interview (ZBI) composto por 22 itens que avaliam as condições de saúde, psicológicas, emocionais, sociais e financeiras que podem vir a ser afetadas em razão dos cuidados prestados ao idoso. Esta escala foi traduzida e validada para a população brasileira em 2002. A pontuação de cada item varia entre 0 e 4 , e o score total da escala, entre 0 e 88 pontos, em que pontuações elevadas indicam altos níveis de sobrecarga. O ponto de corte utilizado para classificação dos cuidadores foram: score $\leq 21$ para ausência de sobrecarga e $\geq 22$ para presença de sobrecarga ${ }^{13}$.

Para relacionar a sobrecarga do cuidador com as características sociodemográficas dos idosos, foram consideradas as seguintes variáveis: idade, sexo, raça, escolaridade, local de residência e quantidade de filhos dos idosos. 


\section{Análise Estatística}

O Teste Chi-quadrado de Pearson ou Exato de Fisher (quando uma ou mais frequências esperadas foi inferior a 5) foi aplicado para comparar as variáveis categóricas do perfil do idoso com a presença ou ausência de sobrecarga nos cuidadores, e o cálculo da Razão de Prevalência foi empregado para as variáveis $\operatorname{com} p<0,10$ na análise inferencial para determinar o tamanho da influência da variável sociodemográfica no desfecho de sobrecarga do cuidador.

A normalidade dos dados contínuos foi verificada utilizando o teste de Kolgomorov-Smirnov. Uma vez identificada a não parametricidade dos dados contínuos, foi aplicado o teste de Correlação de Spearman para determinar a correlação entre o nível de sobrecarga do cuidador com a idade do idoso e com a quantidade de seus filhos. Para interpretação da magnitude da correlação, foi utilizada a seguinte classificação dos coeficientes de correlação: correlação fraca $(<0,4)$; correlação moderada $(\geq 0,4$ a $<0,5)$; e correlação forte $(\geq 0,5)$.

As análises inferenciais foram conduzidas utilizando o software SPSS (IBM 22) e nível de significância de $p<0,05$.

\section{RESULTADOS}

No presente estudo, foram entrevistados 165 cuidadores de idosos restritos ao lar assistidos pela Estratégia Saúde da Família. Verificou-se que a sobrecarga esteve presente em 54 dos entrevistados, o que nos revela uma prevalência de $32,7 \%$ de sobrecarga de cuidadores.

A análise das características sociodemográficas dos idosos com relação à presença ou ausência de sobrecarga dos cuidadores está descrita na tabela 1.

As Razões de Prevalência de sobrecarga de cuidadores com relação a sexo, raça e local de residência dos idosos estão descritas na tabela 2.

A análise da correlação de Spearman evidenciou uma correlação fraca, negativa e estatisticamente significante entre a idade do idoso e a pontuação da ZARIT, ou seja, quanto menor foi a idade do idoso, maior foi a sobrecarga do cuidador. Não foi encontrada correlação entre a sobrecarga de cuidadores e a quantidade de filhos dos idosos. 
Tabela 1. Distribuição das variáveis sócio demográficas dos idosos de acordo com a sobrecarga de seus cuidadores - USF Dr. José Moysés - Vitória/ES

\begin{tabular}{|c|c|c|c|c|}
\hline \multirow[t]{2}{*}{$\begin{array}{c}\text { Variáveis } \\
\text { sociodemográficas } \\
\text { dos Idosos }\end{array}$} & \multirow{2}{*}{$\begin{array}{c}\begin{array}{c}\text { Amostra } \\
(n=165)\end{array} \\
N\end{array}$} & $\begin{array}{c}\text { Ausência de } \\
\text { Sobrecarga de } \\
\text { Cuidadores } \\
(n=111)\end{array}$ & $\begin{array}{c}\text { Presença de } \\
\text { Sobrecarga de } \\
\text { Cuidadores } \\
(n=54)\end{array}$ & \multirow{3}{*}{$p$} \\
\hline & & n $\quad(\%)$ & n $\quad(\%)$ & \\
\hline \multicolumn{4}{|l|}{ Sexo } & \\
\hline Masculino & 39 & $22 \quad(20)$ & $17 \quad(32)$ & \multirow{2}{*}{0,07} \\
\hline Feminino & 126 & $89 \quad(80)$ & $37 \quad(68)$ & \\
\hline \multicolumn{5}{|l|}{$4^{\circ}$ Idade $(>80)$} \\
\hline Sim & 126 & $87 \quad(78)$ & $39 \quad(72)$ & \multirow{2}{*}{0,38} \\
\hline Não & 39 & $24 \quad(22)$ & $15 \quad(28)$ & \\
\hline \multicolumn{5}{|l|}{ Raça } \\
\hline Brancos & 128 & $92 \quad(83)$ & $36 \quad(67)$ & \multirow{2}{*}{$0,019^{*}$} \\
\hline Negros ou Pardos & 37 & $19 \quad(17)$ & $18 \quad(33)$ & \\
\hline \multicolumn{5}{|l|}{ Escolaridade ${ }^{a}$} \\
\hline Analfabetos & 34 & $18 \quad(19)$ & $16 \quad(33)$ & \multirow{5}{*}{0,32} \\
\hline Até 4 anos & 40 & $30 \quad(31)$ & $10 \quad(20)$ & \\
\hline 5 a 8 anos & 33 & $21 \quad(22)$ & $12 \quad(15)$ & \\
\hline 9 a 11 anos & 25 & $17 \quad(18)$ & $8 \quad(16)$ & \\
\hline$>11$ anos & 13 & $10 \quad(10)$ & $3 \quad(6)$ & \\
\hline \multicolumn{5}{|l|}{ Filhos } \\
\hline Sim & 142 & $98 \quad(88)$ & $45 \quad(83)$ & \multirow{2}{*}{0,38} \\
\hline Não & 22 & $13 \quad(12)$ & $9 \quad(17)$ & \\
\hline \multicolumn{5}{|l|}{$\begin{array}{l}\text { Local de } \\
\text { Residência }\end{array}$} \\
\hline Bairro Nobre & 115 & $83 \quad(75)$ & $32 \quad(59)$ & \multirow{2}{*}{$0,04^{*}$} \\
\hline Bairro Popular & 50 & $28 \quad(25)$ & $22 \quad(41)$ & \\
\hline
\end{tabular}


Tabela 2. Razão de Prevalência de sobrecarga em cuidadores de acordo com as características sexo, raça e local de residência de idosos restritos ao lar USF Dr José Moysés - Vitória/ES

\begin{tabular}{|l|c|c|}
\hline \multicolumn{1}{|c|}{$\begin{array}{c}\text { Variáveis } \\
\text { Independentes }\end{array}$} & RP* & IC 95\% \\
\hline Idosos do sexo masculino & 1,48 & $0,95-2,32$ \\
\hline Idosos de raça negra ou parda & 1,72 & $1,12-2,66$ \\
\hline Idosos residentes em bairro nobre & 0,63 & $0,41-0,97$ \\
\hline * RP - Razão de Prevalência & \\
\hline
\end{tabular}

Tabela 3. Distribuição da Correlação de Spearman entre a sobrecarga do cuidador relacionada com a idade e a quantidade de filhos dos idosos restritos ao lar

USF Dr José Moysés - Vitória/ES

\begin{tabular}{|l|c|c|}
\hline \multicolumn{1}{|c|}{ Variáveis Correlacionadas } & Pontuação da Zarit & $\boldsymbol{p}$ \\
\hline Idade do Idoso & $-0,272$ & $<0,001^{*}$ \\
\hline Quantidade de Filhos do Idoso & 0,083 & 0,14 \\
\hline$* p<0,001$ & \\
\hline
\end{tabular}

\section{DIsCUSSÃo}

Neste estudo, identificamos uma prevalência de $32,7 \%$ de sobrecarga nos cuidadores avaliados. $\mathrm{Na}$ literatura, não há uma prevalência determinada para tal desfecho, são vários os estudos encontrados que dissertam sobre a sobrecarga de idosos, entretanto, tais estudos sempre salientam alguma característica determinante nos idosos, como uma doença crônica, sequelas ou dependência funcional. No estudo de Brandão et al. ${ }^{9}$, em que não houve a seleção de cuidadores de idosos com algum tipo de comorbidade, identificou-se uma prevalência de $87,2 \%$ de sobrecarga entre os cuidadores avaliados, porém, apesar de não apresentarem comorbidades específicas, estes idosos eram assistidos por um serviço de atenção domiciliar, em que 48,5\% foram identificados como totalmente dependentes. Já o estudo de Bom, Sá e Cardoso ${ }^{10}$ identificou uma prevalência de $67,9 \%$ de sobrecarga entre os cuidadores formais e informais de idosos de dois centros de atenção à pessoa idosa.

Todavia, independentemente da seleção ou não de um grupo de risco, as prevalências encontradas por este estudo e nos estudos citados diferem consideravelmente. Uma justificativa para o bom resultado encontrado neste estudo pode ser o trabalho desenvolvido pelas equipes de saúde que assistem o território pesquisado. $O$ excesso de carga de trabalho pode ser amenizado com ações 
advindas da Estratégia Saúde da Família, que tem como funções: acompanhar de perto a realidade de cada família; tomar providências para evitar o aparecimento de doenças; atuar para solucionar os casos em que a doença já existe; e prover orientações para garantir uma vida com qualidade e saúde tanto para o idoso como para o cuidador ${ }^{6,14-15}$.

Entretanto, de acordo com estudos de Santos et $\mathrm{al}^{16}$, um dos elementos essenciais para atenção integral do idoso, que propicia a visão integral e ambiental do cuidado prestado a este indivíduo, é a visita domiciliar, um dos grandes problemas para a atenção ao idoso prestado pela Estratégia Saúde da Família. Esses autores pontuam que essa deficiência é de ordem material, com a falta de transporte adequado para os profissionais de saúde, e de recursos humanos, devido à escassez de profissionais especializados e/ou capacitados para essa função. O que é reforçado por estudos de Carvalho, Assunção e Bocchi ${ }^{17}$, ao realizarem uma revisão integrativa sobre a percepção dos profissionais da Estratégia Saúde da Família quanto ao atendimento dispensado aos idosos: eles identificaram que os profissionais reconhecem a falta de preparo da equipe e enfatizam a dificuldade na estrutura organizacional e política da estratégia no que tange à velhice da população.

Quanto às características demográficas dos idosos deste estudo, identificamos um predomínio de idosas do sexo feminino em ambos os grupos, entretanto o grupo com sobrecarga de cuidadores apresentou $12 \%$ mais homens do que o grupo sem sobrecarga, com uma tendência estatística de $p=$ 0,07 . Ressaltando esse achado, encontramos que a prevalência de idosos do sexo masculino foi $48 \%$ maior no grupo de cuidadores com sobrecarga de trabalho (RP 1,48 - IC95\% 0,95 a 2,32). Tais achados corroboram estudos como o de Costa et al. ${ }^{18}$, que também encontraram que uma das características dos idosos que mais sobrecarregam seus cuidadores é ser do sexo masculino.

Os homens apresentam maiores dificuldades para aceitar a necessidade de ser cuidado e a condição de dependência, colaborando para o surgimento de sobrecarga entre os cuidadores. Além disso, preocupam-se menos com a sua saúde o que os tornam mais vulneráveis a agravos de doenças crônicas, o que está ligada diretamente com o comprometimento da autonomia ${ }^{18,19}$. Entretanto, apesar dos achados da literatura e da tendência estatística encontrada neste estudo, esta não é uma informação que pode ser afirmada como verdade.

Não encontramos diferença estatisticamente significante entre os grupos de cuidadores de idosos restritos ao lar com relação a $4^{\circ}$ idade, todavia, a correlação de Spearman identificou que quanto menor foi a idade do idoso, maior foi a sobrecarga do cuidador $(r-0,272-p<0,001)$. Corroborando este resultado, estudos realizados por Costa et al. ${ }^{18}$ e Gratão et al. ${ }^{20}$ mostraram maior ocorrência de sobrecarga em cuidadores de idosos mais jovens.

Esses achados podem estar relacionados com o fato de que o processo senescente em si já coopera para a ocorrência de sobrecarga do cuidador, especialmente se este cuidador for familiar do idoso ${ }^{21}$. Quando se trata de idosos mais novos, que estão acostumados com uma vida independente e autônoma, esse processo pode ser visto com muitas dificuldades e estresse. Somado a isso, temos a ansiedade do cuidador ao ver a impaciência do idoso ao tentar e não conseguir realizar certas tarefas, fazendo, por vezes, com que esse cuidador queira tomar essa responsabilidade para si no 
intento de privar o idoso desse estresse, podendo gerar, então, a sobrecarga física e emocional.

Outra justificativa que pode ser levantada está relacionada com o processo de adaptação. Com o passar do tempo, o cuidador pode estar se adaptando ao cuidado demandado pelo idoso, e não mais se sentir sobrecarregado com as tarefas que, agora, está acostumado a desempenhar.

Com relação à raça, observamos uma grande prevalência de idosos autodeclarados brancos, tanto na população geral quanto nos grupos, porém, no grupo de cuidadores com sobrecarga, houve uma diminuição nessa diferença, com aumento de $13 \%$ de idosos de raça negra ou parda, ou seja, no grupo sem sobrecarga de cuidadores, a prevalência de idosos negros ou pardos é menor $(p 0,019)$. Esse dado se torna ainda mais evidente quando, ao calcular a Razão de Prevalência para esta variável, encontramos que a prevalência de idosos de raça negra ou parda foi $72 \%$ maior no grupo de cuidadores com sobrecarga (RP 1,72 - IC95\% 1,12 a 2,66).

Certo et al. ${ }^{22}$, ao realizarem revisão sistemática da literatura, encontraram que a raça negra é um fator potencializador para o surgimento da fragilidade no idoso, e Cruz et al..$^{23}$, ao analisarem idosos internados em um hospital de Cuiabá, identificaram que a única variável sociodemográfica que foi associada com a capacidade funcional foi a raça, em que os idosos de raça negra apresentaram maior dificuldade para a realização de atividades de vida diária.

De acordo com Certo et al. ${ }^{22}$, a fragilidade é um termo adotado para caracterizar idosos com elevado risco de quedas, hospitalizações e incapacidades; e segundo a Política Nacional de Saúde da Pessoa Idosa ${ }^{24}$, a incapacidade funcional é o principal problema que pode afetar o idoso, visto que esta se caracteriza pela perda das habilidades físicas e psíquicas necessárias para a realização das atividades de vida diária. Dessa forma, é possível entender a associação positiva entre idosos da raça negra/parda com o maior índice de sobrecarga em seus cuidadores.

Quanto ao local de residência dos idosos, encontramos mais idosos residindo em bairros nobres, o que se manteve entre os grupos de cuidadores com e sem sobrecarga. No entanto, identificamos uma diminuição de $16 \%$ na frequência de idosos residentes em bairros nobres no grupo de cuidadores com sobrecarga, sendo esta diferença estatisticamente significante $(p 0,04)$. O cálculo da Razão de Prevalência mostrou que a prevalência de idosos que residem em bairros nobres diminuiu em $37 \%$ no grupo de cuidadores sem sobrecarga (RP 0,63 - IC 95\% 0,41 a 0,97).

A hipótese que pode ser levantada para justificar tal fato é que, residindo em bairros nobres, esses idosos podem ter um poder aquisitivo mais elevado que aqueles que residem em bairros populares. Dessa forma, é possível que seus cuidadores trabalhem em regime formal, ou seja, são cuidadores treinados para a função que exercem; e, dessa forma, apresentam menos chance de desenvolver sobrecarga de trabalho. Bom, Sá e Cardoso ${ }^{10}$, em seu estudo realizado com 53 cuidadores, identificaram que, em sua maioria, os cuidadores formais apresentaram ausência de sobrecarga, em contrapartida, apenas os cuidadores informais apresentaram sobrecarga severa. Entende-se que, sem o devido treinamento, os cuidadores informais - que costumam ser esposas, filhas e netas - estão mais propensos a desenvolver sobrecarga de trabalho. 
No que tange à escolaridade, presença e quantidade de filhos, encontramos um grande número de idosos com baixa escolaridade e com filhos, não havendo diferença estatística para essas variáveis entre os grupos. Também não foi identificada a influência da quantidade de filhos sobre a sobrecarga de cuidadores.

As limitações deste estudo consistem em sua abordagem seccional, que não permite estabelecer relações causais. Além disso, é importante ressaltar que o estudo macro, que deu origem a esta pesquisa, tinha como foco o idoso, fazendo com que os dados do cuidador não tenham sido completamente inquiridos.

\section{CONCLUSÃO}

Foi identificada uma prevalência $32,7 \%$ de sobrecarga de cuidadores; que idosos mais jovens e de raça negra/parda sobrecarregam mais seus cuidadores, enquanto a sobrecarga é menor entre os cuidadores de idosos que residem em bairros nobres.

Acredita-se que os resultados apresentados neste estudo possam servir como base de avaliação diagnóstica, ressaltando a necessidade de enxergar o cuidador como sujeito que também necessita ser cuidado. Ademais, propõe-se que esse cuidado deva ser prestado pelas equipes de saúde da família, em forma de orientações e ações que visem evitar a ocorrência de sobrecarga entre os cuidadores e possibilitar um envelhecimento ativo e saudável aos longevos.

\section{REFERÊNCIAS}

1. World Health Organization. World health statistics 2014: a wealth of information on global public health. Geneva: WHO, 2014.

2. Instituto Brasileiro de Geografia e Estatística. Censo demográfico 2010: características da população e dos domicílios. Rio de Janeiro: IBGE, 2010.

3. Instituto Jones dos Santos Neves. Síntese dos indicadores sociais do Espírito Santo. Vitória: ES, 2013.

4. Araújo I, Paúl C, Martins M. Living older in the family context: dependency in self-care. Rev Esc Enferm USP. 2011;45(4):866-871.

5. Saquetto M, Schettino L, Pinheiro P, Sena ELS, Yarid SD, Gomes Filho DL. Aspectos bioéticos da autonomia do idoso. Rev Bioét. 2013;21(3):518-524.

6. Ministério da Saúde (Brasil). Secretaria de Atenção à Saúde. Departamento de Ações Programáticas e Estratégicas. Área Técnica Saúde do Idoso. Guia prático do cuidador. Brasília: Ministério da Saúde, 2. ed. 2009.

7. Coutinho AT, Popim RC, Carregã K, Spiri WC. Integrality of care for the aged in the family health strategy: the vision of the team. Esc Anna Nery. 2013 Sep-Dec;17(4):628-637.

8. Frutuoso JT, Cruz RM. Mensuração da carga de trabalho e sua relação com a saúde do trabalhador. Rev Bras Med Trab [Internet] 2005 [acessado 2018 mar 25]; 3(1):29-36. Disponível em: www.rbmt.org.br/ export-pdf/166/v3n1a05.pdf.

9. Brandão FSR, Costa BGS, Cavalcanti ZR, Bezerra MR, Alencar LCA, Leal MCC. Overload of elderly people caregivers assisted by a home care service. J Nurs UFPE on line. 2017;11(Suppl. 1):272-279. DOI: http://dx.doi.org/10.5205/reuol.7995-69931-4-SM.1101sup201704.

10. Bom FS, Sá SPC, Cardoso RSS. Overload in caregivers of the elderly. J Nurs UFPE on line. 2017;11(1):160-164 
11. Stackfleth R, Diniz MA, Fhon JRS, Vendruscolo TRP, Coelho SC, Fabrício-Whebe SCC et al. Burden of work in caregivers of frail elders living at home. Acta paul. Enferm. 2012;25(5):768-774.

12. Ursine PGS, Cordeiro HA, Moraes CL. Prevalência de idosos restritos ao domicílio em região metropolitana de Belo Horizonte (Minas Gerais, Brasil). Ciênc Saúde Col. 2011;16(6):2953-2962.

13. Scazufca M. Brazilian version of the Burden Interview scale for the assessment of burden of care in carers of people with mental illnesses. Rev Bras Psiquiatr.2002;24(1):12-17.

14. Ministério da Saúde (Brasil). Política nacional de atenção básica - PNAB. Brasília, DF: Ministério da Saúde, 2012.

15. Martins JJ, Albuquerque GL, Nascimento ERP, Barra DCC, Souza WGA, Pacheco NSP. Necessidades de educação em saúde dos cuidadores de pessoas idosas no domicílio. Texto Contexto Enferm. 2007;16(2):25462.

16. Santos CTB, Andrade LOM, Silva MJ, Souza MF. Percurso do idoso em redes de atenção à saúde: um elo a ser construído. Physis. 2016;26(1):45-62.

17. Carvalho CJA, Assunção RC, Bocchi SCM. Percepção dos profissionais que atuam na Estratégia de Saúde da Família quanto à assistência prestada aos idosos: revisão integrativa da literatura. Physis. 2010;20(4):1307-1324.

18. Costa TF, Costa KNFM, Martins KP, Fernandes MGM, Brito SS. Burden over family caregivers of elderly people with stroke. Esc Anna Nery. 2015;19(2):350-355.

19. Baptista BO, Beuter M, Girardon-Perlini NMO, Brondani CM, Budó MLD, Santos NO. A sobrecarga do familiar cuidador no âmbito domiciliar: uma revisão integrativa da literatura. Rev Gaúcha Enferm. 2012;33(1):147-56.

20. Gratão ACM, Talmelli LFS, Figueiredo LC, Rosset I, Freitas CP, Rodrigues RAP. Functional dependency of older individuals and caregiver burden. Rev Esc Enferm USP. 2013;47(1):134-41.

21. Andrade LM, Costa MFM, Caetano JA, Soares E, Beserra EP. The problematic aspects of the family caregiver of people who suffered strokes. Rev Esc Enferm USP. 2009;43(1):35-40.

22. Certo AC, Sanchez K, Galvão A, Fernandes H. A síndrome da fragilidade nos idosos: revisão da literatura. Actas de Gerontologia [Internet] 2016 [acessado 2018 mar 25]; 2(1):1-11. Disponível em http:// actasdegerontologia.pt/index.php/Gerontologia/article/view/56.

23. Cruz APM, Pinto RLC, Lage YG, Nasrala MLS, Alterações da Capacidade Funcional de Idoso Durante a Internação Hospitalar. Coorte [Internet] 2011 [acessado 2018 mar 25]; 3(3):22-29. Disponível em http:// revistacoorte.com.br/index.php/coorte/article/view/18/37.

24. Ministério da Saúde (Brasil). Portaria $n^{\circ} 2.528$, de 19 de outubro de 2006. Institui a Política Nacional de Saúde da Pessoa Idosa. Diário Oficial da União 20 nov 2006. 